\title{
The value of transthoracic and transesophageal echocardiography for the diagnosis of the native aortic infective endocarditis valve complications: a case report and literature review.
}

\author{
Adrian Molnar ${ }^{1}$, Diana Sacui ${ }^{1}$, Simona Manole ${ }^{2}$, Amanda Radulescu ${ }^{3}$, Ruxandra Beyer ${ }^{4}$ \\ ${ }^{1}$ Cardiovascular Surgery Department, Heart Institute, ${ }^{2}$ Radiology Clinic, Emergency Clinic County Hospital, ${ }^{3}$ Infec- \\ tious Disease Clinic, ${ }^{4}$ Cardiology Department, Heart Institute, Cluj-Napoca, Romania
}

\begin{abstract}
Despite its current limitations transthoracic echocardiography is still widely used for the anatomical and functional evaluation of patients with infective endocarditis. However, all these limitations can be overcome by using transesophageal echocardiography. We present the case of a 42 -year-old male patient, diagnosed with aortic valve infective endocarditis, whose transthoracic echocardiography showed only a cusp vegetation and aortic regurgitation, but raised the suspicion of periannular complications. The transesophageal echocardiography revealed a circular aortic root abscess and a ventricular septal defect with left-to-right shunt, and consequently leads to a complete different surgical tactical approach. The patient was urgently referred for surgery due to the rapid deterioration of the hemodynamic status, and had a good outcome on the short-term follow-up.

Keywords: infective endocarditis, echocardiography, periannular complications
\end{abstract}

\section{Introduction}

Infective endocarditis (IE) remains a cardiac pathology associated with high morbidity and mortality, and furthermore a major challenge for the cardiac surgeon [1]. Despite the improving quality of life as well as the evolving diagnosis methods and antibiotic therapy, IE continues to affect a relatively large segment of the active population with an incidence of 3-10 episodes/100 000 person-years (mostly men aged between 70 and 80 years) [2-4].

Depending on the site of infection and the presence or absence of intracardiac foreign material, IE is classified as follows: left-sided native valve IE, left-sided prosthetic valve IE, right-sided IE, and device-related IE

Received 10.10.2015 Accepted 16.11.2015

Med Ultrason

2016, Vol. 18, No 2, 253-256

Corresponding author: Diana Sacui

Cardiovascular Surgery Clinic, Heart Institute 19-21, Motilor street,

400001, Cluj-Napoca, Romania

Phone: +40-264-591.941

E-mail: diana_sacui27@yahoo.com
(IE developed on pacemaker or defibrillator wires, with or without the involvement of the valve). Regarding the acquisition, IE is divided into community-acquired IE, health care-associated IE (nosocomial and non-nosocomial), and IE in intravenous drug abusers (IVDAs) [2]. The new European Society of Cardiology (ESC) guidelines emphasize the role of transthoracic echocardiography (TTE) as a first line imaging study in the diagnosis of this disease (Class IB recommendation) [5].

Transesophageal echocardiography (TEE) is a noninvasive and cost-effective examination, able to comprehensively characterize the morphology and functional status of the cardiac and vascular structures affected by the infectious process. This information is essential in the setting of the surgical approach, considering that a favorable long term outcome depends on the complete removal of the infected tissue followed by the reconstruction or replacement of the excised lesions. Sometimes these excisions are widely extended, and the tissue destruction is extremely large. In these situations, the diagnostic accuracy and the magnitude of surgical reconstruction become a challenge for both the cardiologist and the cardiac surgeon such as the case we are going to presentnow. 


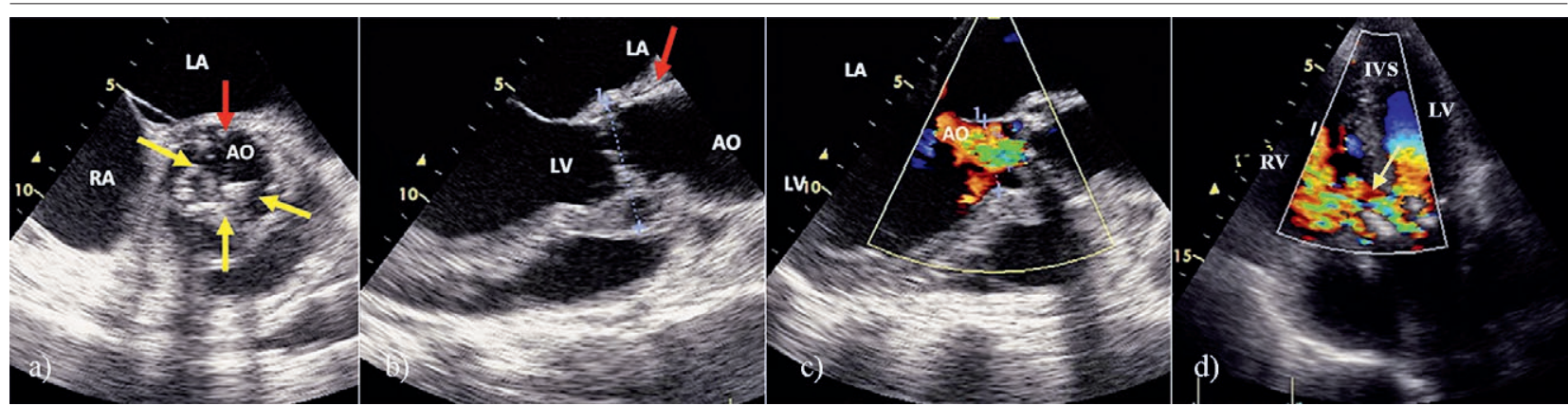

Fig 1. a) Transesophageal short axis view of the aortic valve: vegetation (inhomogeneous structures attached to the valvular tissue), perforations, retractions, and scars (white arrows) were found. In the periannular region between the aorta and left atrium an echolucent space suggests the presence of an aortic root abscess (red arrow); b) Transesophageal long axis view of the aortic valve and ascending aorta. The echolucent space located around the aortic valve annulus and proximal ascending aorta suggests a circular aortic root abscess (red arrows); c) Transesophageal long axis view of the aortic valve and ascending aorta, colour Doppler. Severe aortic regurgitation caused by a co-aptation defect of the modified and perforated cusps; d) Transthoracic echocardiography, apical 4-chamber view, colour Doppler. A colour flow from the aortic root into the right ventricle indicates a left-to-right shunt caused by a fistula between the aortic root and the right ventricle (arrow). LV: left ventricle; RV: right ventricle; IVS: interventricular septum; Ao: ascending aorta.

\section{Case report}

A 42-year-old man was admitted to our intensive care unit for dyspnea at rest and extreme malaise. The patient had a 4-week history of fever, anorexia, and $6 \mathrm{~kg}$ loss weight. He denied any history of travelling, prior surgery, dental procedures, or contact with animals over the previous 3 months.

One week before presentation he was admitted to the Infectious Disease Clinic. Physical examination revealed that time a dehydrated patient with hyperpyrexia $\left(42^{\circ} \mathrm{C}\right)$, blood pressure of $80 / 60 \mathrm{mmHg}$ and heart rate 104 beats/ minute. Blood tests showed elevation of $\mathrm{C}$-reactive protein to $16.7 \mathrm{mg} / \mathrm{dL}$ (normal values: $<1 \mathrm{mg} / \mathrm{dL}$ ), increase of the white blood cells count to $20,400 / \mu \mathrm{L}$ and normocytic anemia with a hemoglobin level of $11.8 \mathrm{~g} / \mathrm{dL}$. In all three sets of blood cultures obtained in the first hours after admission (30 minutes apart), Gram staining detected Gram-positive diplococci - Streptococcus pneumonia with sensitivity to penicillin G. Chest radiography revealed peribronchial reticulonodular opacity. TTE was carried out using a GE Vivid S6 echo system, which illustrated a $0.6 \mathrm{~cm}$ vegetation attached to the right coronary cusp of the aortic valve and moderate aortic regurgitation (vena contracta $5 \mathrm{~mm}$, pressure half time PHT $550 \mathrm{~ms}$, velocity time integral VTI in the descending aorta $6 \mathrm{~cm}$ ). No periannular complications were detected.

According to Duke criteria [6], the patient was diagnosed with definite infective endocarditis; subsequently, antibiotherapy with Ceftriaxone and Gentamycin was initiated. One week after admission to the Infectious Disease Clinic, the patient had an unfavorable clinical course with an ongoing high fever, worsening of dysp- nea, fatigue and hypotension, and consequently he was transferred to our emergency department. At admission the electrocardiogram revealed sinus tachycardia, right bundle branch block, and right axis deviation. The chest X-ray showed a slightly dilated left heart and pulmonary edema. Due to the high probability of periannular complications, a transesophageal echocardiography (TEE) was performed (GE Vivid S6 echo system). The bi-dimensional (2D) images identified a tricuspid aortic valve with severe endocarditic lesions: vegetation, cusp ruptures, and retractions. The echolucent space located around the aortic valve annulus and proximal ascending aorta suggested a circular aortic root abscess. Additionally, a severe aortic regurgitation (vena contracta $12 \mathrm{~mm}$, PHT $160 \mathrm{~ms}$, VTI in the descending aorta $20 \mathrm{~cm}$ ) was identified. In the apical 4-chamber view a continuous color flow from the aortic root into the right ventricle indicated a perforation in the aortic wall, erosion into the right ventricle and creation of a continuous left-to-right shunt between the aortic root and the right ventricle, suggesting the presence of a fistula (fig 1). The presence of the shunt at the level of the basal interventricular septal region suggested the extension of the infectious process to the membranous septum, with an important ventricular septal defect (VSD). The left and right ventricles were enlarged (LV diameters 65/38 mm, RV diameter $45 \mathrm{~mm}$ below tricuspid valve), with hyperdynamic contraction and normal systolic function (LVEF 68\%, TAPSE 30 $\mathrm{mm}$ ). Also a moderate pulmonary hypertension (PASP $60 \mathrm{mmHg}$ ) was found. The patient was referred for emergency cardiac surgery

Intraoperatively purulent pericardial liquid and thick pericardial layers, with inflammatory changes were 


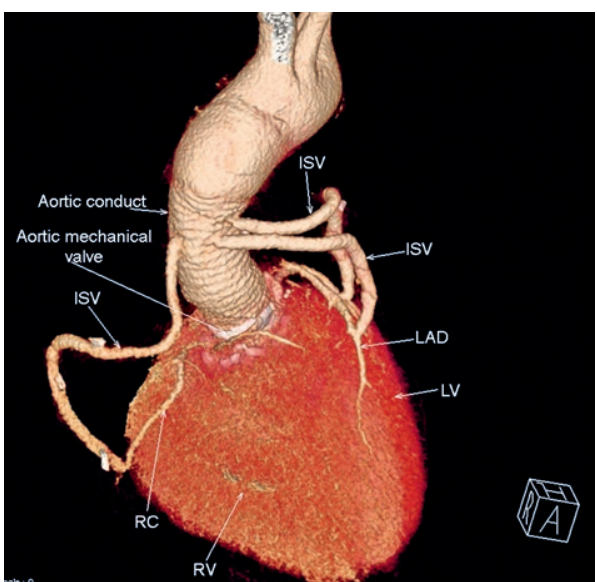

Fig 2. Cardiac computed tomography (3-D reconstruction), 1 month after surgery: aortic conduit containing a mechanical prosthesis and triple coronary artery bypass graft. All grafts are patent, without complications and without peri-prosthetic effusions or false aneurysms. ISV: internal saphenous vein; RC: right coronary (artery); RV: right ventricle; LV: left ventricle; LAD: left anterior descending (artery).

found. On the anterior aortic wall a tumor mass of approximately $4 / 3 \mathrm{~cm}$ was observed and was interpreted as an aortic root abscess. The inspection of the aortic valve revealed a $2 / 2 \mathrm{~cm}$ vegetation on the right coronary commissure, a small abscess on the left coronary commissure, and a giant abscess with a fistula opened into the right ventricle, lying between the left and right coronary ostia, with a large VSD. The muscular and the aortic walls were extremely friable, and a small fistula between the inner and the outer aortic layers on the anterior aortic wall was also noticed. The aortic intima was completely damaged, with severe inflammatory lesions at the level of the coronary ostia. The infected aortic valve and the surrounding necrotic tissue were excised. The VSD was covered with bovine pericardial patch on the inferior wall, and continuous suture of the other walls. A valved conduit containing a mechanical prosthesis of $23 \mathrm{~mm}$ and a tubular Dacron graft of $25 \mathrm{~mm}$ was inserted. The re-implantation of the coronary ostia into the tubular graft was technically not possible so a triple coronary bypass (CABG) with saphenous veins was performed. Intraoperative TEE was used to control the success of the procedure.

The overall postoperative course of the patient was uneventful. Two weeks postoperatively, TTE was repeated. The aortic mechanic prosthesis was with normal pressure gradients. There was no communication between the aortic root and heart chambers and no residual interventricular shunt. In order to evaluate the postoperative results, a CT-angiography of the aorta was performed 3 months after surgery (fig 2).

\section{Discussions}

Considering the main risk factors for IE of the native valve (increased longevity, prosthetic valves, increased exposure to nosocomial bacteremia, use of iv drugs, HIV infection, mitral valve prolapsed) [7-10] we found no risk factors for IE in our patient. As perivalvular IE is usually associated with a higher incidence of serious complications, a more complicated surgical procedure, or death, early diagnosis is essential [11]. Useful information concerning the diagnosis and the severity of IE, as well as the short- and long-term prognosis are given by TEE [12].

In our case, neither the initial nor the second TTE performed by a very experienced cardiologist were able to detect other lesions than the vegetation attached to the right coronary cusp of the aortic valve with secondary moderate aortic regurgitation. The presence of the periannular abscess and the VSD with left-to-right shunt were revealed only by the TEE. Our results are in line with literature data [13], confirming that TEE was an essential tool in our patient. In these circumstances, we can state that TEE had a conclusive role for the comprehensive preoperative diagnosis. The accuracy of the images provided by this technique was confirmed by the intraoperative findings. Moreover the ESC guideline recommends to perform TEE not only pre-operatively for diagnosis purposes, but also intraoperatively in all cases of infective endocarditis requiring surgery, due to its ability its affect at least one of the following parameters operative plan, quality control of valve repair/replacement, hemodynamic assessment or de-airing [5,14]. Precise recognition of possible complications is important for the medical and surgical management of these patients, as those complications could generate unexplained congestive heart failure and hemodynamic deterioration in some cases with aortic valve endocarditis [14]. Extension of the infection to the perivalvular tissues is a sign of poor prognosis in the evolution of the disease: it may lead to endothelial erosion, perivalvular abscess, mycotic aneurysm, and intracardiac fistulae [12]. Intraoperative findings in our patient were characterized by severe damage at the level of the aortic wall, coronary ostia and inter-ventricular septum; these supplementary lesions made the surgical technique more complicated and challenging.

The risk of recurrence among survivors of IE varies between 2.7 and $22.5 \%$ and prophylactic measures should be very strict, involving postoperative regular echocardiography control examinations (TTE, TEE) [2]. We used TEE immediately after finishing the surgical procedure and TTE two weeks post-operatively in order to have the best evaluation of our patient's outcome, besides the significant clinical improvement. These non- 
invasive techniques allowed us to be confident with the complex treatment (surgical and medical) managed for this patient.

The repetition of TTE and TEE in the patients' follow-up program is extremely important, as it is stressed in the 2015 ESC guidelines: TTE is recommended at completion of antibiotic therapy for evaluation of cardiac and valve morphology and function [5].

Finally, we would like to emphasize once more the case complexity, with regard to the imaging studies (TTE, TEE, and CT-angiography), as well as regarding the surgical technique (aortic root reconstruction, ventricular septal defect closure, aortic valve replacement and triple $\mathrm{CABG}$ ). Another particularity would be the relatively rare etiology (Pneumococcus), but with high severity and mainly encountered in young patients.

In conclusion the complete and accurate preoperative diagnosis in IE and their optimal surgical corrections remain challenging. In the presence of clinical manifestations suggestive for IE, all patients should undergo TTE. In all cases with positive results a TEE is mandatory, in order to identify possible complications for an adequate surgical timing.

\section{References}

1. Prendergast BD, Tornos P. Surgery for infective endocarditis: who and when? Circulation 2010; 121: 1141-1152.

2. Habib G, Hoen B, Tornos P, et al. Guidelines on the prevention, diagnosis, and treatment of infective endocarditis (new version 2009): the Task Force on the Prevention, Diagnosis, and Treatment of Infective Endocarditis of the European Society of Cardiology (ESC). Endorsed by the European Society of Clinical Microbiology and Infectious Diseases (ESCMID) and the International Society of Chemotherapy (ISC) for Infection and Cancer. Eur Heart J 2009; 30: 2369-2413.

3. Baddour LM, Wilson WR, Bayer AS, et al. Infective endocarditis: diagnosis, antimicrobial therapy, and management of complications: a statement for healthcare professionals from the Committee on Rheumatic Fever, Endocarditis, and Kawasaki Disease, Council on Cardiovascular Disease in the Young, and the Councils on Clinical Cardiology, Stroke, and Cardiovascular Surgery and Anesthesia, American Heart Association: endorsed by the Infectious Diseases Society of America. Circulation 2005; 111: e394-e434.

4. Hoen B, Duval X. Infective endocarditis. N Engl J Med 2013; 369: 785.

5. Habib G, Lancellotti P, Antunes MJ, et al. 2015 ESC Guidelines for the management of infective endocarditis. The Task Force for the Management of Infective Endocarditis of the European Society of Cardiology (ESC) Endorsed by: European Association for Cardio-Thoracic Surgery (EACTS), the European Association of Nuclear Medicine (EANM)]. Eur Heart J 2015; 36: 3075-3128.

6. Habib G, Badano L, Tribouilloy C, et al. Recommendations for the practice of echocardiography in infective endocarditis. Eur J Echocardiogr 2010; 11: 202-219.

7. Thalme A, Nygren AT, Julander I, Freyschuss U. Classification of infective endocarditis by Duke's criteria and transesophageal echocardiography: a 1-year retrospective analysis. Scand J Infect Dis 1996; 28: 407-410.

8. Strom BL, Abrutyn E, Berlin JA, et al. Risk factors for infective endocarditis: oral hygiene and nondental exposures. Circulation 2000; 102: 2842-2848.

9. Calderwood SB, Swinski LA, Waternaux CM, Karchmer AW, Buckley MJ. Risk factors for the development of prosthetic valve endocarditis. Circulation 1985; 72: 31-37.

10. Mylonakis E, Calderwood SB. Infective endocarditis in adults. N Engl J Med 2001; 345: 1318-1330.

11. Taams MA, Gussenhoven EJ, Bos E, et al. Enhanced morphological diagnosis in infective endocarditis by transoesophageal echocardiography. Br Heart J 1990; 63: 109-113.

12. Flachskampf FA, Wouters PF, Edvardsen T, et al. Recommendations for transoesophageal echocardiography: EACVI update 2014. Eur Heart J Cardiovasc Imaging 2014; 15 : 353-365.

13. Evangelista A, Gonzalez-Alujas MT. Echocardiography in infective endocarditis. Heart 2004; 90: 614-617.

14. Karalis DG, Bansal RC, Hauck AJ, et al. Transesophageal echocardiographic recognition of subaortic complications in aortic valve endocarditis. Clinical and surgical implications. Circulation 1992; 86: 353-362. 